\title{
An improved sequence based prediction protocol for DNA-binding proteins using SVM and comprehensive feature analysis
}

\author{
Chuanxin Zou, Jiayu Gong and Honglin Li
}

\begin{abstract}
Background: DNA-binding proteins (DNA-BPs) play a pivotal role in both eukaryotic and prokaryotic proteomes. There have been several computational methods proposed in the literature to deal with the DNA-BPs, many informative features and properties were used and proved to have significant impact on this problem. However the ultimate goal of Bioinformatics is to be able to predict the DNA-BPs directly from primary sequence.

Results: In this work, the focus is how to transform these informative features into uniform numeric representation appropriately and improve the prediction accuracy of our SVM-based classifier for DNA-BPs. A systematic representation of some selected features known to perform well is investigated here. Firstly, four kinds of protein properties are obtained and used to describe the protein sequence. Secondly, three different feature transformation methods (OCTD, AC and SAA) are adopted to obtain numeric feature vectors from three main levels: Global, Nonlocal and Local of protein sequence and their performances are exhaustively investigated. At last, the mRMR-IFS feature selection method and ensemble learning approach are utilized to determine the best prediction model. Besides, the optimal features selected by mRMR-IFS are illustrated based on the observed results which may provide useful insights for revealing the mechanisms of protein-DNA interactions. For five-fold cross-validation over the DNAdset and DNAaset, we obtained an overall accuracy of 0.940 and 0.811 , MCC of 0.881 and 0.614 respectively.

Conclusions: The good results suggest that it can efficiently develop an entirely sequence-based protocol that transforms and integrates informative features from different scales used by SVM to predict DNA-BPs accurately. Moreover, a novel systematic framework for sequence descriptor-based protein function prediction is proposed here.
\end{abstract}

\section{Background}

DNA binding proteins (DNA-BPs) that interact with DNA are pivotal to the cell function such as DNA replication, transcription, packaging recombination and other fundamental activities associated with DNA. DNA-BPs represent a broad category of proteins, known to be highly diverse in sequence, structure and function. Structurally, they have been divided into eight structural/functional groups, which were further classified into 54 structural families [1]. Functionally, protein-DNA interactions play various roles across the entire genome as previously mentioned [2]. At present, several experimental techniques (such as filter binding assays, genetic analysis, chromatin

\footnotetext{
* Correspondence: hlli@mail.shcnc.ac.cn

Shanghai Key Laboratory of New Drug Design, State Key Laboratory of Bioreactor Engineering, School of Pharmacy, East China University of Science and Technology, Shanghai 200237, China
}

immunoprecipitation on microarrays, and X-ray crystallography) have been used for identifying DNA-BPs. However, experimental approaches for identifying the DNA-BPs are costly and time consuming. Therefore, a reliable identification of DNA-BPs as well as DNA-binding sites with effective computational approach is an important research topic in the proteomics fields, which can play a vital role in proteome function annotation and discovery of potential therapeutics for genetic diseases and reliable diagnostics.

Computational prediction of proteins that interact with DNA is a difficult task, and state of the art methods have shown only limited success in this arena at present. Previously, there have been several machine-learning methods developed for prediction of DNA-BPs in the literature. Broadly, these methods can be divided into two categories: i) analysis from protein structure [3-6] and ii)

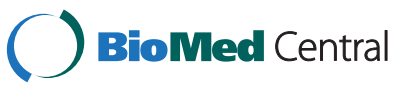

(c) 2013 Zou et al.; licensee BioMed Central Ltd. This is an Open Access article distributed under the terms of the Creative Commons Attribution License (http://creativecommons.org/licenses/by/2.0), which permits unrestricted use, distribution, and reproduction in any medium, provided the original work is properly cited. 
prediction from amino acid sequence [7-12]. The accuracy of structure-based prediction methods is usually higher, but it can't be used in high throughput annotation with the limited number of protein structures. Theoretically, the sequence of a protein contains all the necessary information to predict its function [13]. Until now, many methods for predicting protein function directly from amino acid sequences are useful tools in the study of uncharacterized protein families and in comparative genomics [14]. There are two major problems in the task of computational protein function prediction, which are the choice of the protein representation and the choice of the classification algorithm. To obtain good predictive model, various machine-learning algorithms such as support vector machine (SVM) [8,10,15-18], neural network $[3,6,19]$, random forest $[12,20]$, naïve Bayes classifiers [21,22], nearest neighbor [23] and ensemble classifiers $[24,25]$ have been used to build classification models. Among these, the most widely used algorithm for prediction of DNA-BPs is SVM.

In context to the current study, SVM learns the features specific to the DNA-BPs and generates support vectors decisive for possible classification of any given sequence as DNA-BPs and achieved satisfactory results. The most important challenge for SVM-based prediction is to find a suitable way to fully describe the information implied in protein-DNA interactions [26]. There are several different protein features and feature extraction methods that can be used [8,27-29] and a comprehensive survey of these methods can be found in related research work [30,31]. However, the underlying principle of proteinDNA interactions is still largely unknown. It is desirable to explore the implications of those already identified features and newly undiscovered properties by machine learning approaches to further advance the prediction accuracy and understand the binding mechanism of DNA-BPs.

Thus, a systematic comparison of all protein features known to perform well is investigated in this article. We propose a novel method for predicting DNA-BPs using the SVM algorithm in conjunction with comprehensive feature analysis based on protein sequence. A recent work about mechanisms of protein folding research [32] has shown that the property factors of protein can be naturally clustered into two classes. One class is comprised of properties that favor sequentially localized interaction clusters; the other class is in favor of globally distributed interactions. Following the methodology introduced earlier in related protein function prediction work [29-31], we consider a feature vector (xi) to represent proteins which are derived from sequences broadly from three main levels: Global sequence descriptors, Nonlocal sequence descriptors and Local sequence descriptors. Feature vectors extracted from different sequence levels contain information about characteristics of the proteins at different scales which may be helpful in describing the information implied in protein-DNA interactions and improving the final model accuracy.

This paper consists of three main parts: Firstly, we investigate four different kinds of protein features which are composition information, structural and functional information, physicochemical properties and evolutionary information derived from reported literatures, public databases, and related prediction systems. The most informative and representative features are roughly derived from these four kinds of properties. Secondly, three different coding methods are adopted to represent different protein features selected above. These methods are called OCTD (Global method), auto covariance (AC) (Nonlocal method) and SAA (Local method). Lastly, the performance of different feature extraction strategies are extensively investigated by individual SVM classifiers, we collect and analyze those descriptors generated by means of good prediction behaviors. The mRMR-IFS feature selection approach and ensemble learning method are adopted to determine the ultimate prediction model. Our results show that accurate prediction of DNA-BPs can be obtained using a comprehensive analysis of global, nonlocal and local information of protein sequence together. The overall workflow of our method is shown in Figure 1.

\section{Methods \\ Datasets}

Four types of datasets are used here: i) DNAdset consists of partial sequences (binding regions or DNA binding domains) ii) DNAaset consists of full-length DNA-binding proteins. It's reported that models trained on DNA domains or partial sequences are not suitable for predicting DNA binding proteins and vice versa, so separate methods are necessary for predicting DNA-binding domains and DNAbinding proteins [10]. iii) An independent test set called DNAiset used for testing and comparing. iiii) DNArset with non-equal number of positives and negatives used for evaluating our method in real life.

\section{DNAdset}

The domain dataset also called DNAdset, consists of 231 DNA-BPs and 231 non-binding proteins with known structures which were obtained from a union of datasets used in previously related studies $[8,9,16]$. After clustering by CD-HIT [33] and careful inspection, these proteins have less than $40 \%$ sequence identity between each pair and without irregular amino acid characters such as " $X$ " and " $Z$ ". Thus the obtained DNAdset consists of 462 proteins, half of which are DNA-BPs and the other half are non-binding proteins. A complete list of all the PDB codes for DNAdset can be found in Additional file 1. 


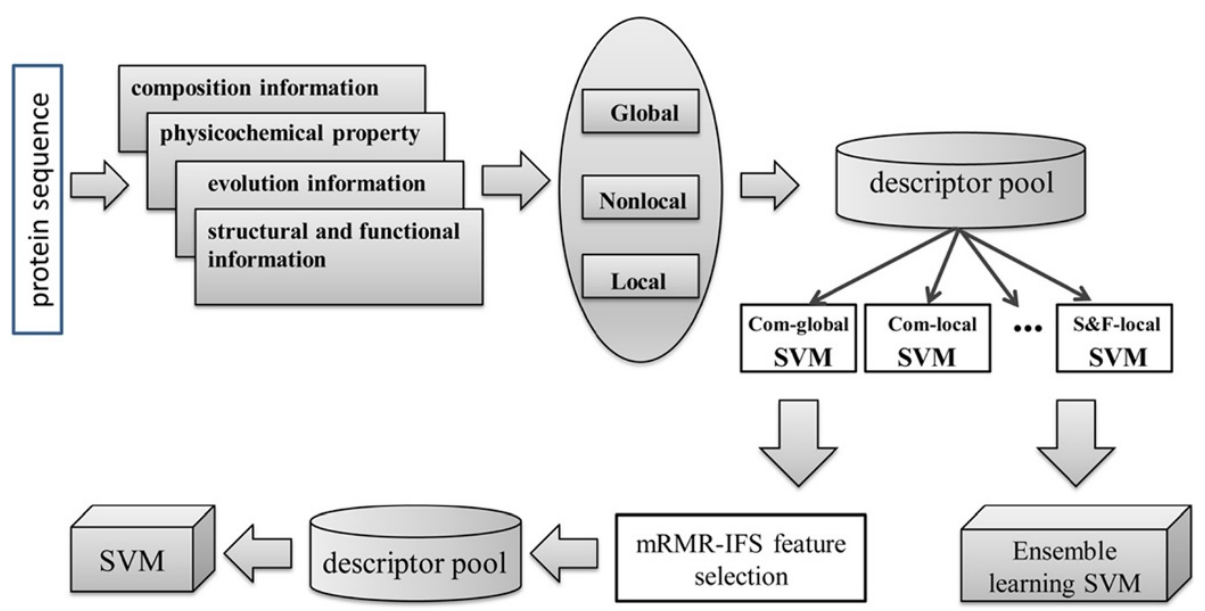

Figure 1 The overall workflow of the present method. Firstly, the input amino acid sequence is represented numerically by four kinds of features. Secondly, these feature values are transformed to feature descriptor matrices from three different levels. Thirdly, the first round of the evaluation is adopted based on the original descriptor pool and individual SVM models obtained. At last, mRMR-IFS feature selection method and ensemble learning approach are applied as the final evaluation of the optimal SVM model.

\section{DNAaset}

To evaluate effectiveness of our methods by comparing with previously famous studies, we used the benchmark DNAaset from reported papers [10,17]. The dataset consists of 1153 DNA-binding proteins and 1153 non-binding proteins obtained from Swiss-Prot. No two protein sequences have similarity more than $25 \%$ and without irregular amino acid characters such as " $\mathrm{X}$ " and " $\mathrm{Z}$ ".

\section{DNAiset}

In order to evaluate performance of our models on dataset not used for training or testing and compare with other reported methods, we obtained an independent dataset called DNAiset from newly determined DNA-binding protein structures from PDB by keyword searching (released on 2012-01-01 and later) and non-binding proteins used in a reported prediction method [12]. To reduce the redundancy and homology bias, CD-HIT [33] and PISCES [34] programs were used to ensure no two protein sequences have similarity more than $30 \%$ between DNAiset and two training sets (i.e. DNAdset and DNArset). Finally, the DNAiset has 80 DNA-binding protein chains selected from PDB and 192 non-binding proteins obtained from a newly developed web server named iDNA-Prot [12]. A complete list of all the PDB codes for DNAiset can be found in Additional file 1.

\section{DNArset}

Equal number of positives and negatives is important for developing a powerful predictor for a protein system. It's also important for evaluating the performance of the prediction model where one can simply calculate the accuracy. All the above datasets in our study have equal number of DNA-binding proteins and non- binding proteins. However, in a real-world situation, DNA-binding proteins are only a fraction of all proteins. It's one of the (relatively) new problems called imbalanced dataset which has received an increasing attention since the workshop at AAAI 2000 [35]. This raises questions on whether models developed on equal numbers will be effective in real life. Will the method have a significantly poorer performance with more negatives in a test case? Thus, we created a more realistic dataset called DNArset to answer it. This dataset has 231 DNA-BPs used in DNAdset and 1500 non-binding proteins used by Kumar et al. as their "DNArset" [10].

\section{Support vector machine}

Support vector machine (SVM) is a machine learning algorithm based on statistical learning theory presented by Vapnik (1998). It takes a set of feature vectors as the input, along with their output, which is used for training of model. Application of SVM in bioinformatics to various topics has been explored [16]. In this study, publicly available LIBSVM package version 3.11 [36] is used for the implementation of SVM and the RBF is taken as the kernel function, the tunable parameters are optimized based on grid search method to deliver high accuracy. All feature descriptors derived below were normalized in the range of $[0,1]$ by using formula (value-minimum)/ (maximum-minimum) before training SVM.

\section{Protein features}

To develop a powerful function predictor for a protein system, one of the keys is to formulate the datasets with an effective mathematical expression that can truly reflect their intrinsic correlation with the attribute to be predicted [27]. To realize this, we assess four kinds of 
features including composition information, physicochemical property, evolutionary information and structural/ functional property. The feature vector representations of these features are generated from three different levels, including Global sequence descriptors, Nonlocal descriptors and Local descriptors. Here, the composition information including overall amino acid composition (global descriptors), Dipeptide composition (nonlocal descriptors) and split amino acid composition (local descriptors). The other three kinds of properties are transformed by three different feature transformation methods which are introduced detailedly in Methods section.

\section{Overall amino acid composition (OAAC)}

The conventional overall amino acid composition is defined as a 20-dimensional vector, which consists of the occurrence frequencies of 20 native amino acids. Given a protein $P$ :

$$
p_{i}=\frac{n_{i}}{L} \quad(i=1,2 \ldots, 20)
$$

Where $p_{i}$ represents the occurrence frequency of the $i$-th native amino acid in the protein, $n_{i}$ is the number of the $i$-th native amino acid in sequence, $L$ is the length of the sequence in protein $P$.

It is reported that better performance could be obtained by calculating the square root of $p_{i}$ instead [37]. The algorithm was inspired by the principle of superposition of state in quantum mechanics and proved good here. So we use $f_{i}$ as overall amino acid composition (OAAC) information.

$$
f_{i}=\sqrt{p_{i}} \quad(i=1,2 \ldots, 20)
$$

\section{Dipeptide composition (DPC)}

Dipeptide composition (DPC) comprises of two consecutive residues which gives a fixed pattern length of 400. This widely used sequence representation encapsulates information about the fraction of amino acids as well as their local order [38]. In this paper, firstly, three kinds of Dipeptide composition $D P_{O}, D P_{1}, D P_{2}$ are calculated by counting all pairs of amino acid condition with
0, 1 and 2 skips respectively [22] as shown in Figure 2. Each kind of Dipeptide composition gives 400 descriptors, defined as:

$$
f_{s}(i, j)=\frac{D_{s}(i, j)}{N-1} \quad(i, j=1,2,3, \ldots, 20 \quad s=0,1,2)
$$

Where $D_{s}(i, j)$ is the number of Dipeptide represented by amino acid type $i$ and $j$ with $s$ skips, $f_{s}(i, j)$ represents the occurrence frequency. $N$ is the length of the sequence.

Then, we concatenate the vector elements of $D P_{0}, D P_{1}$ and $D P_{2}$ together and the mRMR method [39] is adopted to select the first 400 descriptors from the total of 1200 dimensions as DPC used in this paper.

\section{Split amino acid composition (SAAC)}

Split Amino Acid Composition (SAAC) was introduced where the protein sequence is divided into three parts: $\mathrm{N}$ terminal, $\mathrm{C}$ terminal and a region between them and composition of each part is calculated separately according to equations (1) and (2). Many previous literatures adopted SAAC for protein function prediction and achieved good results $[40,41]$. In our SAAC method, the detailed strategy of splitting the protein sequence is illustrated in Split amino acid (SAA) Transformation section.

\section{Physicochemical properties}

Physicochemical properties of amino acids have been successfully employed in many sequence based function predictions with characteristics of well defined and high interpretability $[17,40,42]$. AAIndex [43] is a well known database of amino acid biochemical and physicochemical properties. Recently, a systematic approach (named AutoIDPCPs) has been conducted to identify informative physicochemical and biochemical properties in the AAIndex database to design SVM-based classifiers for predicting and analyzing DNA-BPs [17]. We use the selected 28 representative numerical index scores in their paper to encode each amino acid in this study, as shown in Table 1. A complete protein sequence is then represented by a set

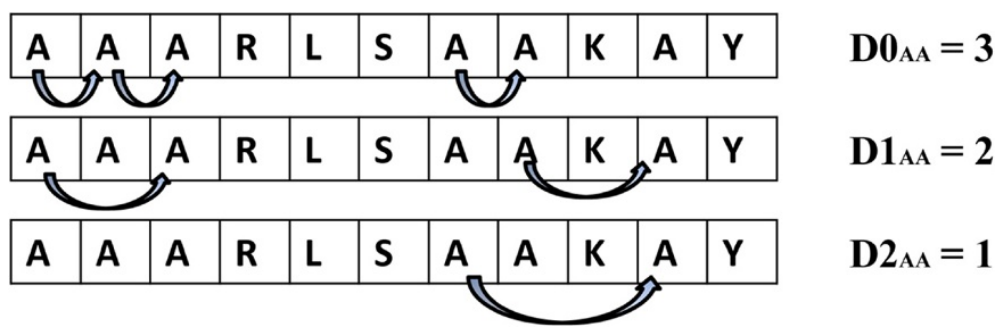

Figure 2 The count of three kinds of Dipeptide composition D0, D1, D2. 
Table 1 List of the AAlndex indices used in this paper

\begin{tabular}{|c|c|c|}
\hline Feature ID & AAIndex ID & Feature description \\
\hline 39 & CHOP780202 & $\begin{array}{l}\text { Normalized frequency of beta-sheet } \\
\text { (Chou-Fasman, 1978b) }\end{array}$ \\
\hline 56 & $\mathrm{CIDH} 920103$ & $\begin{array}{l}\text { Normalized hydrophobicity scales for } \\
\text { alpha+beta-proteins (Cid et al., 1992) }\end{array}$ \\
\hline 58 & $\mathrm{CIDH} 920105$ & $\begin{array}{l}\text { Normalized average hydrophobicity } \\
\text { scales (Cid et al., 1992) }\end{array}$ \\
\hline 86 & FAUJ880109 & $\begin{array}{l}\text { Number of hydrogen bond donors } \\
\text { (Fauchere et al., 1988) }\end{array}$ \\
\hline 88 & FAUJ880111 & Positive charge (Fauchere et al., 1988) \\
\hline 95 & FINA910104 & $\begin{array}{l}\text { Helix termination parameter at posision } \\
\text { j+1 (Finkelstein et al., 1991) }\end{array}$ \\
\hline 100 & GEIM800104 & $\begin{array}{l}\text { Alpha-helix indices for alpha/beta- } \\
\text { proteins (Geisow-Roberts, 1980) }\end{array}$ \\
\hline 102 & GEIM800106 & $\begin{array}{l}\text { Beta-strand indices for beta-proteins } \\
\text { (Geisow-Roberts, 1980) }\end{array}$ \\
\hline 139 & KANM800102 & $\begin{array}{l}\text { Average relative probability of beta- } \\
\text { sheet (Kanehisa-Tsong, 1980) }\end{array}$ \\
\hline 146 & KLEP840101 & Net charge (Klein et al., 1984) \\
\hline 147 & KRIW710101 & $\begin{array}{l}\text { Side chain interaction parameter } \\
\text { (Krigbaum-Rubin, 1971) }\end{array}$ \\
\hline 167 & LIFS790101 & $\begin{array}{l}\text { Conformational preference for all beta- } \\
\text { strands (Lifson-Sander, 1979) }\end{array}$ \\
\hline 178 & MEEJ800101 & $\begin{array}{l}\text { Retention coefficient in HPLC, pH7.4 } \\
\text { (Meek, 1980) }\end{array}$ \\
\hline 214 & OOBM770102 & $\begin{array}{l}\text { Short and medium range non-bonded } \\
\text { energy per atom (Oobatake-Ooi, 1977) }\end{array}$ \\
\hline 229 & PALJ810107 & $\begin{array}{l}\text { Normalized frequency of alpha-helix in } \\
\text { all-alpha class (Palau et al., 1981) }\end{array}$ \\
\hline 280 & QIAN880123 & $\begin{array}{l}\text { Weights for beta-sheet at the window } \\
\text { position of } 3 \text { (Qian-Sejnowski, 1988) }\end{array}$ \\
\hline 299 & RACS770103 & $\begin{array}{l}\text { Side chain orientational preference } \\
\text { (Rackovsky-Scheraga, 1977) }\end{array}$ \\
\hline 321 & RADA880108 & $\begin{array}{l}\text { Mean polarity } \\
\text { (Radzicka-Wolfenden, 1988) }\end{array}$ \\
\hline 356 & ROSM880102 & $\begin{array}{l}\text { Side chain hydropathy, corrected for } \\
\text { solvation (Roseman, 1988) }\end{array}$ \\
\hline 365 & SWER830101 & $\begin{array}{l}\text { Optimal matching hydrophobicity } \\
\text { (Sweet-Eisenberg, 1983) }\end{array}$ \\
\hline 399 & ZIMJ680102 & Bulkiness (Zimmerman et al., 1968) \\
\hline 401 & ZIMJ680104 & $\begin{array}{l}\text { Isoelectric point } \\
\text { (Zimmerman et al., 1968) }\end{array}$ \\
\hline 422 & AURR980120 & $\begin{array}{l}\text { Normalized positional residue } \\
\text { frequency at helix termini C4' } \\
\text { (Aurora-Rose, 1998) }\end{array}$ \\
\hline 431 & MUNV940103 & $\begin{array}{l}\text { Free energy in beta-strand } \\
\text { conformation (Munoz-Serrano, 1994) }\end{array}$ \\
\hline 449 & NADH010104 & $\begin{array}{l}\text { Hydropathy scale based on self- } \\
\text { information values in the two-state } \\
\text { model ( } 20 \% \text { accessibility) } \\
\text { (Naderi-Manesh et al., 2001) }\end{array}$ \\
\hline 451 & NADH010106 & $\begin{array}{l}\text { Hydropathy scale based on } \\
\text { self-information values in the two- } \\
\text { state model ( } 36 \% \text { accessibility) } \\
\text { (Naderi-Manesh et al., 2001) }\end{array}$ \\
\hline
\end{tabular}

Table 1 List of the AAIndex indices used in this paper (Continued)

\begin{tabular}{lll}
\hline 512 & GUYH850105 & $\begin{array}{l}\text { Apparent partition energies calculated } \\
\text { from Chothia index (Guy, 1985) }\end{array}$ \\
528 & MIYS990104 & $\begin{array}{l}\text { Optimized relative partition energies - } \\
\text { method C (Miyazawa-Jernigan, 1999) }\end{array}$ \\
\hline
\end{tabular}

of $28 \times L$ numerical strings, each of which records the course of one property value along the $L$-residue sequence.

\section{PSSM profiles}

To use the evolution information, the position-specific scoring matrix (PSSM) [44] profiles are adopted, which have been widely used in protein function prediction and other bioinformatics problems with notable improvement of performance $[10,45]$. Here, the PSSM profiles are generated by using the PSI-Blast program [44] to search the non-redundant (NR) database (released on 14. May 2011) through three iterations with 0.001 as the E-value cutoff for multiple sequence alignment. The final PSSM scoring matrix has $20 \times L$ elements (excluding dummy residue $\mathrm{X}$ ), where $L$ is the length of protein.

\section{Secondary structure composition}

Secondary structure is an important structural feature of protein that can significantly improve the function prediction performance $[46,47]$. In this study, secondary structure calculation is carried out by PSIPRED v3.0 [48], which is one of the state-of-the-art protein secondary structure prediction methods with an accuracy of up to $80 \%$. PSIPRED predicts secondary structure for each residue in a protein and provides a confidence score for three types of secondary structures: helices, $\beta$-sheets and coil regions, thus we get $3 \times L$ feature values where $L$ is the length of protein.

\section{Disorder feature score}

Over the past decade, there has been a growing acknowledgement that a large proportion of proteins within most proteomes contain disordered regions. Protein with disordered regions can play important functional roles. Its flexibility is advantageous to proteins that recognize multiple target molecules including biomacromolecules like DNA with high specificity and low affinity [49-51]. The IUPred [52] method is used to score the disorder status of each amino acid which recognizes intrinsically disordered protein regions from amino acid sequences by estimating their total pairwise interresidue interaction energy. The prediction type option for IUPred is set as long and we get $L$ feature values where $L$ is the length of protein. 


\section{Feature transformation method}

Three different methods are used here to transform preselected protein features into uniform length descriptors to capture various types of information implied in proteins. The following part describes in detail the methodology for each of these different transformation methods.

\section{Overall composition-transition-distribution (OCTD)}

The original CTD method was first introduced by Dubchak et al. [53] as a global description of protein sequence for predicting protein folding class. Recently, CTD has been adopted by more and more leading investigators for protein function and structure studies [54,55] . Composition $(\mathrm{C})$ is the number of amino acids of a particular property divided by the total number of amino acids. Transition $(\mathrm{T})$ characterizes the percent frequency with which amino acids of a particular property is followed by amino acids of a different property. Distribution (D) measures the chain length within which the first, 25, 50, 75 and 100\% of the amino acids of a particular property is located respectively. Here, we develop a new variant method of CTD named Overall Composition-Transition-Distribution (OCTD). Initially, we represent the sequence numerically for a particular feature. Then, we normalize the feature values in the range of $[0,1]$ using formula (valueminimum)/ (maximum-minimum), amino acids were grouped into two classes according to its feature values of threshold 0.5. Finally, the CTD method is adopted to represent the amino acid properties distribution pattern of a specific property along the protein sequence.

\section{Autocross-covariance (ACC) Transformation}

The Autocross-covariance (ACC) method is a simplified nonlocal statistical tool for analyzing sequences of vectors which developed by Wold et al. [56]. Recently, ACC has been adopted by many protein function prediction studies [57,58] including DNA-BPs [8]. ACC method results in two kinds of variables, $\mathrm{AC}$ between the same kind of descriptors, and cross covariance (CC) between two different descriptors. In this study, only AC variables are used in order to avoid generating too large number of descriptors and based on the observations from previously related studies [58]. AC variables describe the average interactions between two residues, a certain $l g$ apart throughout the whole sequence. Here, $l g$ is the distance between one residue and its neighbor, a certain number of residues away. The $\mathrm{AC}$ variables are calculated according to Equation (4) below:

$$
A C_{(i, \lg )}=\sum_{j=1}^{L=\lg }\left(S_{i, j}-\bar{S}_{i}\right)\left(S_{i, j+\lg }-\bar{S}_{i}\right) /(L-\lg )
$$

Where $i$ is one of the properties, $j$ is the position in the sequence, $L$ is the length of the protein sequence $S_{i, j}$ is the feature value of $i$ at position $j, \bar{S}_{i}$ is the average score for amino acid $i$ along the whole sequence:

$$
\bar{S}_{i}=\sum_{j=1}^{L} S_{i, j} / L
$$

Thus, the number of $\mathrm{AC}$ variables can be calculated as $P \times L G$, where $P$ is the number of feature value, $L G$ is the maximum of $\lg (\lg =1,2, \ldots, L G)$.

\section{Split amino acid (SAA) Transformation}

There have been several ways to calculate protein local features, the proposed method split amino acid composition (SAAC) where composition of N-terminal, middle and C-terminal of protein is computed separately [40,41]. The PNPRD method which divided the protein sequence into 10 local regions based on positively and negatively charged residues [59] is some kind of variation from CTD method. There are also more concise method by splitting each protein into 10 local regions of varying length and CTD method was used to exact descriptors from local regions $[60,61]$.

Here, we adopt the powerful split amino acid (SAA) method to represent local composition of different protein features selected before. Firstly, each sequence is split into three parts: $\mathrm{N}$-terminal, middle and $\mathrm{C}$-terminal. The N-terminal part is further divided into four regions to consider ambiguity in the length and position of signal sequences. Then, the mean of different features corresponding to the six divided local regions are obtained to generate a fixed number of local descriptors.

We define the $\mathrm{N}$-terminal, middle and $\mathrm{C}$-terminal parts depending on sequence length $L$. The $\mathrm{N}$-terminal part is further divided into four regions with length $d_{N}$ which is set to 25. It's also assumed that the middle part $d_{\mathrm{M}}$ has at least 20 residues equal to the number of distinct amino acids. The length of the $\mathrm{C}$-terminal part $d_{\mathrm{C}}$ is set to 10 (Figure 3A). For short sequences, we use two more definitions. If $L$ is $>4 d_{\mathrm{N}}+d_{\mathrm{C}}$ and $<4 d_{\mathrm{N}}+20+d_{\mathrm{C}}$, the middle part is regarded as 20 residues from the start of the C-terminal part toward the $\mathrm{N}$-terminal part (Figure 3B). In the case that $L$ is $\leq 4 d_{\mathrm{N}}+d_{\mathrm{C}}$, we assumed that the lengths of the $\mathrm{N}$-terminal and middle parts are the same which are equal to $\left(L-d_{C}\right) / 2$ and the $\mathrm{N}$ terminal part is not divided at all (Figure 3C).

\section{Features selection}

Maximum Relevance, Minimum Redundancy (mRMR) method was first proposed by Peng for processing microarray data [39]. Max-Relevance means that candidate feature to be selected preferentially has the maximal correlation with the target variable, while MinRedundancy requires that candidate feature to be selected preferentially has minimal redundancy with the 

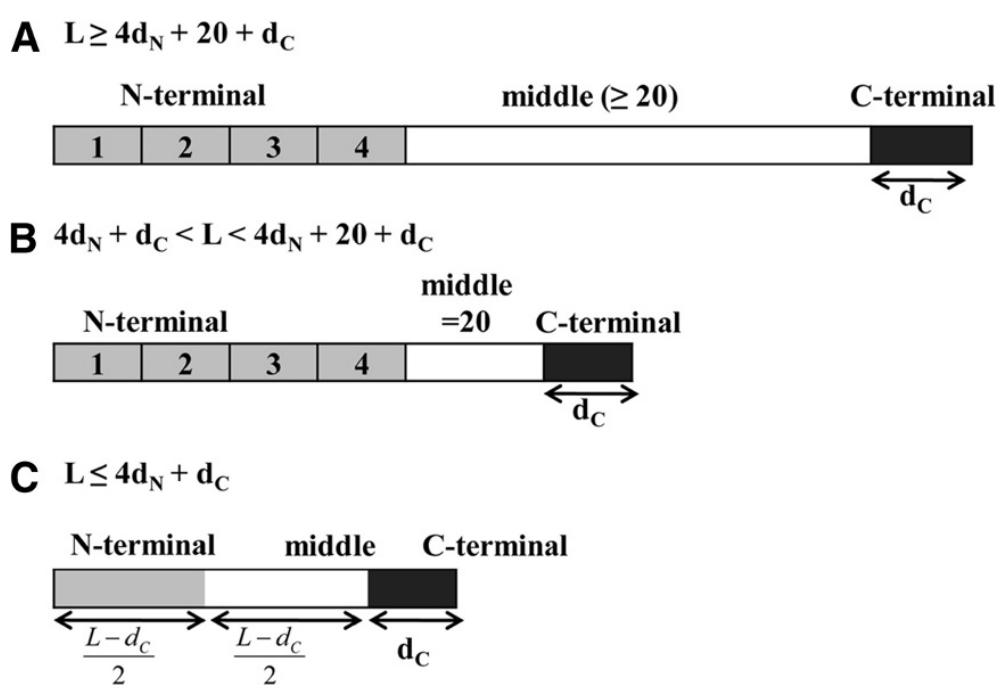

Figure 3 Definitions of the N-terminal, middle, and C-terminal parts depending on sequence length $L$ for SAA method.

features already selected. Both Relevance and Redundancy are measured by the mutual information (MI) defined as:

$$
I(x, y)-\iint p(x, y) \log \frac{p(x, y)}{p(x) p(y)} d x d y
$$

Where $x$ and $y$ are two random variables, $p(x, y)$ is the joint probabilistic density, $p(x)$ and $p(y)$ are the marginal probabilistic densities respectively. The mathematical description of the algorithm was detailedly presented in the Peng's previous study [39]. To calculate MI, the joint probabilistic density and the marginal probabilistic densities of the two vectors were used. A parameter $t$ is introduced here to deal with these variables. Suppose mean to be the average value of one feature in all samples, and std to be the standard deviation, the feature of each sample would be classified into one of the three groups according to the boundaries: mean \pm ( $t \times s t d)$. Here, $t$ was assigned to be 1 .

After mRMR procedure, mRMR feature pool $S$ (shown as eq(7)) containing all features in an ordered way is obtained. Now we know the advantages of the features, but we do not know how many features and which features we should choose. Incremental Feature Selection (IFS) step was utilized to determine the optimal number of features and the optimal features based on mRMR method.

$$
S=\left\{f_{0}, f_{1}, \ldots, f_{h}, \ldots, f_{N-1}\right\}
$$

Incremental Feature Selection (IFS) According to mRMR result, we can construct the $N$ feature sets from ordered feature set $\boldsymbol{S}(\mathrm{eq}(7))$ as follows:

$$
S_{i}=\left\{f_{0}, f_{1}, \ldots, f_{1}\right\} \quad(0 \leq i \leq N-i)
$$

Where $f_{i}$ is the $i$-th sorted feature in the feature list.

For each feature subset, we use SVM to construct predictor which is evaluated by five-fold cross-validation. As a result, we get a curve named IFS curve, with MCC value as its $y$-axis and index $i$ of $S_{i}$ as its $x$-axis. When the overall IFS curve reaches at the peak, meanwhile, the corresponding predictor is chosen as the ultimate prediction model.

\section{Ensemble learning method}

The idea of ensemble learning methodology is to build a predictive model by integrating multiple models, treating them as a committee of decision makers. As a growing body of studies indicates that every single learning strategy has its own shortcomings and none of them could consistently perform well over all datasets. To overcome this problem, ensemble methods have been suggested as the promising measures [62,63]. In general, an ensemble consists of a set of models and a method to combine them. We have twelve different SVM models after the first round of evaluation for exploring the performance of SVM-based modules constructed by different types of features (see Results section for details). Two popular model combination strategies: majority voting and stacking are adopted here.

In majority voting scheme, a classification of an unlabeled instance is performed according to the predicted class that obtains the highest number of votes. That is, we have twelve different classifiers in this work, if a majority of the twelve modules predict a protein as binding, then the prediction result of this protein is taken as binding. When equal number occurred, we found most proteins in this situation are non-binding so the threshold is assigned to six for binding prediction in majority voting scheme.

Stacking is a technique for achieving the highest generalization accuracy [25,63]. Different from the majority voting scheme, stacking will learning twice. The basic idea 
is to learn a function that combines the predictions from the individual classifiers. Instead of using the original input attributes, it uses the predicted probabilities for every class label from the base-level classifiers as the input attributes of the target for the second-level machine learning. In our work, we use the decision values from the twelve SVM modules as the input feature vectors for final meta SVM-predictor in stacking scheme.

\section{Performance evaluation}

Six parameters are employed to indicate the performance of our method, including overall accuracy (Acc), area under the receiver operating characteristic curve (AUC), Fscore, sensitivity (Sen), specificity (Sp) and Matthews's correlation coefficient (MCC). Details of these indices are listed in Table 2. The five-fold cross validation method is used to evaluate model which can minimize the overfitting of the prediction model, the whole dataset is randomly separated into five parts. Each time, one part is retained for testing and all others form the training dataset. This process is repeated five times to test each subset. The evaluation parameters above are calculated as the average from the 5-fold cross validation.

\section{Results}

AC results

To evaluate the AC transformation method, we first check the impact of parameter $L G$ to achieve the best characterization of the protein sequence. Here, we use both Acc and AUC parameters as optimization objectives to determine the optimal values of $L G$ for each feature transformation. The performance of different AC transformed features for different values of $L G$ on the DNAdset and DNAaset is shown in Figure 4. To make a uniform representation, the optimal values of $L G$ for AAindex-AC, PSSM-AC and S\&F-AC (S\&F means the Structural and Functional information which include Secondary Structure Composition and Disorder Feature Score) are set to 9, 11 and 10 respectively in both DNAdset and DNAaset.

Table 2 Indices used to evaluate the prediction method

\begin{tabular}{|c|c|}
\hline Index & Definition and formula \\
\hline Acc & $(T P+T N) /(T P+T N+F P+F N)$ \\
\hline$\overline{A \cup C}$ & area under the receiving operating characteristic curve \\
\hline F-score & $2 \cdot T P /(2 T P+F P+F N)$ \\
\hline Sen & $T P /(T P+F N)$ \\
\hline$\overline{S p}$ & $T N /(T N+F P)$ \\
\hline MCC & $\frac{T P \cdot T N-F N \cdot F P}{\sqrt{(T P+F N) \cdot(T P+F P) \cdot(T N+F N) \cdot(T P+F P)}}$ \\
\hline
\end{tabular}

TP (true positive); TN (true negative); FP (false positive); FN (false negative); Acc, overall accuracy; AUC, area under ROC; Sen, sensitivity; Sp, specificity; F-score, $2 \times$ precision×sensitivity/(precision+sensitivity); MCC, Matthews's correlation coefficient.

\section{Individual SVM-modules results}

The detailed procedure of our method is illustrated in Figure 1. The details about the selected properties of protein sequence and how the feature descriptor matrices were compiled are outlined in Methods section. During the first round of the evaluation, we explore the performance of SVM-based modules constructed by different features transformed from various levels. They are termed OAAC, DPC, SAAC, AAIndex-OCTD, AAIndex-AC, AAIndexSAA, PSSM-OCTD, PSSM-AC, PSSM-SAA, S\&F-OCTD, S\&F-AC and S\&F-SAA respectively. The prediction results of individual SVM-modules using 5-fold cross-validation over the DNAdset and DNAaset are tabulated in Table 3. As shown in Table 3, most of these descriptors are well performed in discriminating the DNA-BPs from non-DNA -BPs as expected. The reason is that those carefully selected informative features are well performed ones in previous studies or some inherent properties related to protein-DNA interactions. Moreover, it demonstrates the usefulness of feature transformation methods used here. Sequence composition information is the basic characters of protein sequences and modules based on the three kinds of composition descriptors are well performed as expected. For predicting DNAaset, PSSM profiles transformed by SAA method are more powerful than commonly used PSSM400 [10] which has an accuracy of $74.22 \%$ and MCC of 0.49. The performances of PSSM-OCTD and PSSM-AC are equal to PSSM-400 method. There is no much difference among three transformation methods for S\&F feature, but moderate performances in both DNAdset and DNAaset are noticeable not only because of the difference between the two datasets but also because of the limited accuracy of PSIPRED and IUPred which is just around $80 \%$. So we believe that protein secondary structure and disorder patterns are closely connected with the process of proteinDNA interactions and some related papers have discussed on it $[49,64]$. In the future, we believe the prediction power of combined S\&F features will significantly improve when more accurate prediction methods of secondary structure and disorder patterns are developed. For AAIndex features transformed by OCTD method, the Acc parameter is 0.743 , a little less than the Auto-IDPCPs method which used the same 28 AAIndex [17] with an accuracy of 0.755 . The poor performance of the nonlocal descriptors AAIndex-AC is somewhat unexpected as we speculated that the coding method should be able to capture some information of amino acid physicochemical and biochemical properties related to the nonlocal nature. However, previous research has demonstrated the existence of only two types of physicochemical and biochemical properties which are locally and globally distributed [32]. So, we are continuing to investigate the implications of these observations.

After investigating individual coding scheme, it's confirmed that all twelve kinds of descriptors are reasonable 

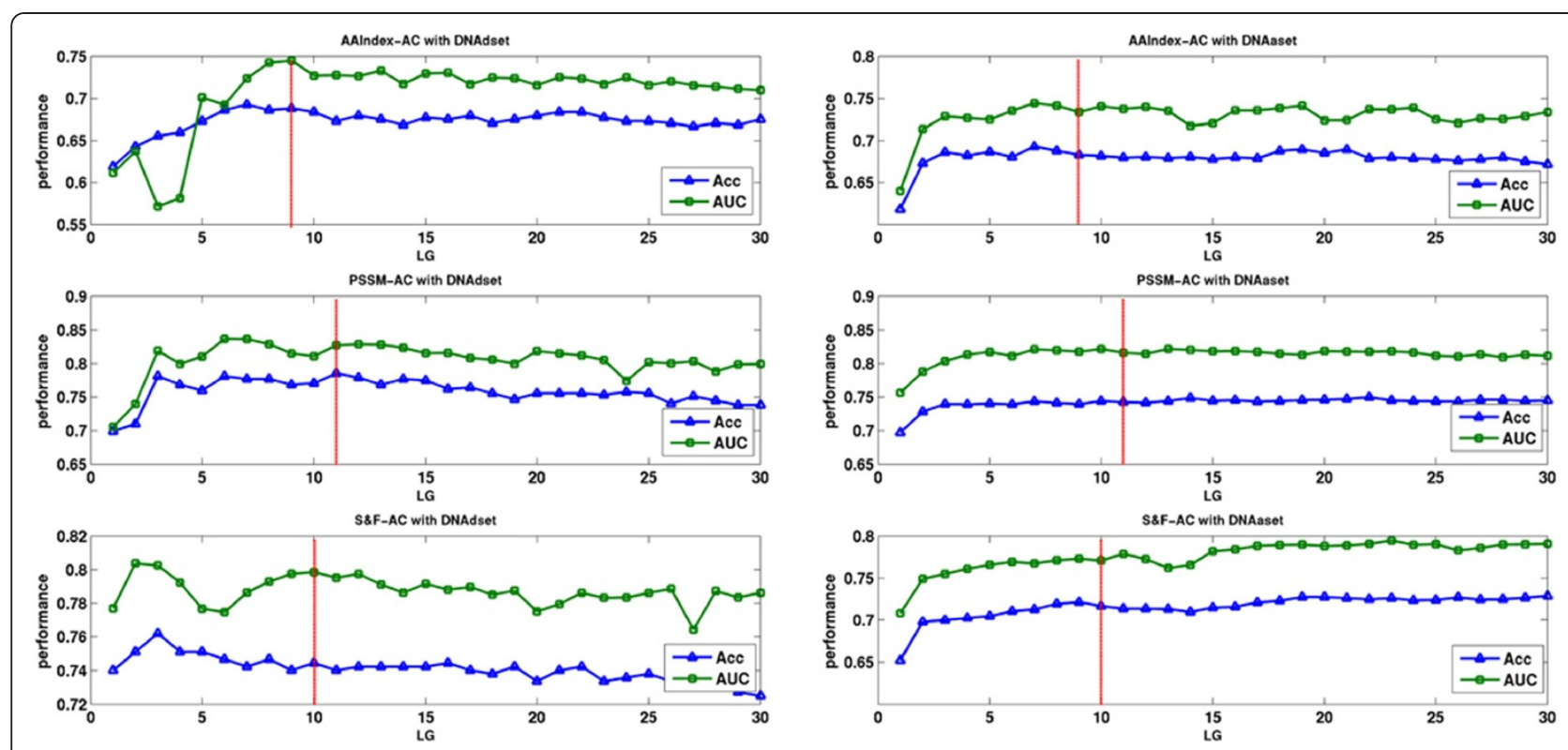

Figure 4 The performance of different AC features with various $L G$ values over DNAdset and DNAaset.

for discriminating DNA-BPs. We intend to combine the well performed descriptors above-mentioned which mean a comprehensive presentation of protein functional features related to DNA binding. Thus, two different strategies are proposed here for achieving this goal which are ensemble learning and feature selection.

To achieve the reduction of noise and redundancy to improve the classification accuracy and the combination of more interpretable features that can help identify DNA-BPs, the proposed mRMR-IFS feature selection framework is adopted.

\section{MRMR results}

The mRMR program in this study is downloaded from http://penglab.janelia.org/proj/mRMR/. Using the mRMR program, we obtain the ranked mRMR list of the first 1000 features from the original 2040 descriptors for DNAdset and DNAaset separately. Within the list, a smaller index of a feature indicates that it is deemed as a more important feature in the prediction. The mRMR list is retained and will be used in the IFS procedure for feature selection and further analysis.

\section{IFS results}

On the basis of the outputs of mRMR, we built individual predictors by adding features recursively from the top of the mRMR list to the bottom. As shown in Figure 5, the plotted IFS curve and the detailed IFS results can be found in Additional file 2 and Additional file 3. As we can see, the maximum MCC is 0.881 (accuracy is 0.940 ) with 203

Table 3 The performance of different kinds of feature descriptors

\begin{tabular}{|c|c|c|c|c|c|c|c|c|c|c|c|c|}
\hline \multirow[t]{2}{*}{ Descriptor } & \multicolumn{6}{|c|}{ DNAdset } & \multicolumn{6}{|c|}{ DNAaset } \\
\hline & Acc & AUC & F-score & Sen & Sp & MCC & Acc & AUC & F-score & Sen & Sp & MCC \\
\hline OAAC & 0.872 & 0.941 & 0.856 & 0.865 & 0.852 & 0.716 & 0.726 & 0.794 & 0.742 & 0.799 & 0.650 & 0.451 \\
\hline $\mathrm{DPC}$ & 0.872 & 0.925 & 0.838 & 0.865 & 0.809 & 0.672 & 0.717 & 0.784 & 0.725 & 0.753 & 0.682 & 0.436 \\
\hline SAAC & 0.846 & 0.904 & 0.826 & 0.842 & 0.813 & 0.651 & 0.697 & 0.740 & 0.701 & 0.743 & 0.624 & 0.369 \\
\hline AAlndex-OCTD & 0.845 & 0.905 & 0.824 & 0.828 & 0.825 & 0.651 & 0.743 & 0.782 & 0.729 & 0.766 & 0.664 & 0.452 \\
\hline AAindex-AC & 0.688 & 0.745 & 0.707 & 0.729 & 0.680 & 0.410 & 0.683 & 0.734 & 0.705 & 0.785 & 0.559 & 0.353 \\
\hline AAlndex-SAA & 0.870 & 0.915 & 0.840 & 0.869 & 0.811 & 0.678 & 0.708 & 0.747 & 0.732 & 0.808 & 0.601 & 0.417 \\
\hline PSSM-OCTD & 0.729 & 0.776 & 0.721 & 0.728 & 0.724 & 0.452 & 0.741 & 0.811 & 0.742 & 0.745 & 0.738 & 0.483 \\
\hline PSSM-AC & 0.786 & 0.827 & 0.762 & 0.771 & 0.752 & 0.523 & 0.742 & 0.816 & 0.734 & 0.725 & 0.751 & 0.477 \\
\hline PSSM-SAA & 0.872 & 0.932 & 0.872 & 0.903 & 0.839 & 0.741 & 0.761 & 0.840 & 0.773 & 0.797 & 0.737 & 0.535 \\
\hline S\&F-OCTD & 0.723 & 0.801 & 0.737 & 0.714 & 0.779 & 0.493 & 0.719 & 0.770 & 0.711 & 0.726 & 0.694 & 0.411 \\
\hline S\&F-AC & 0.745 & 0.799 & 0.729 & 0.756 & 0.690 & 0.446 & 0.717 & 0.771 & 0.701 & 0.690 & 0.723 & 0.413 \\
\hline S\&F-SAA & 0.712 & 0.734 & 0.649 & 0.627 & 0.736 & 0.371 & 0.711 & 0.768 & 0.703 & 0.710 & 0.692 & 0.402 \\
\hline
\end{tabular}




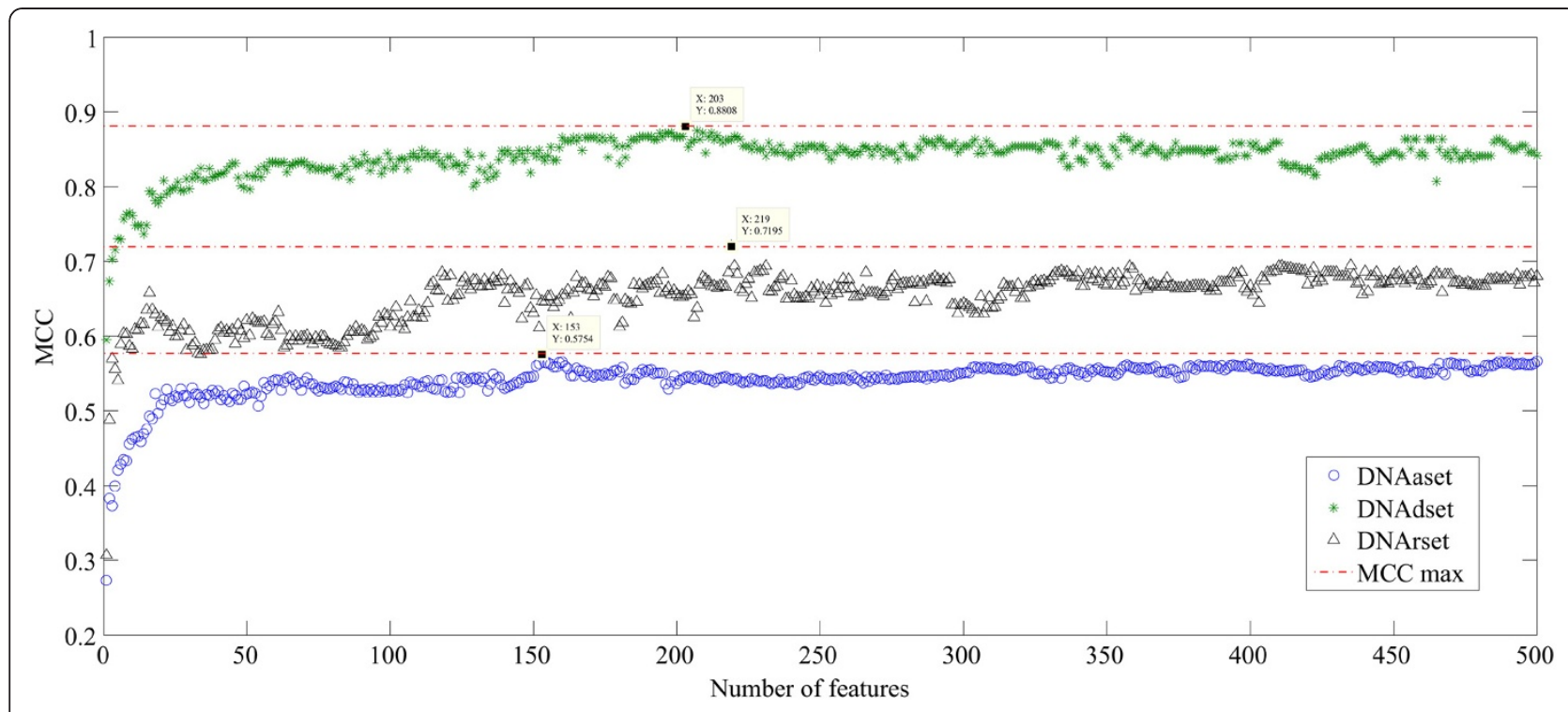

Figure 5 The IFS curves of DNAdset, DNArset and DNAaset.

features and 0.575 (accuracy is 0.789) with 153 features for DNAdset and DNAaset respectively. So, these selected features are considered as the optimal feature set used in our final prediction model.

\section{Ensemble learning results}

Based on the results of individual SVM-modules, ensemble learning method attempts to combine different models into a consensus classifier by majority voting and stacking method. The performance of ensemble learning with different combining method is listed in Table 4. As shown in Tables 3 and 4, the ensemble learning results are much better than those individual meta predictors including the excellent component classifier such as PSSM-SAA and DPC. Among the two kinds of combining method, stacking (with accuracy of 0.907 and 0.811 for DNAdset and DNAaset) outperforms majority voting (with accuracy of 0.898 and 0.789 respectively) which may benefit from the discrimination power of SVM and the idea of divideand-conquer.

\section{Performance on independent dataset}

In this study, we further evaluated the performance of our optimal SVM models (trained on DNAdset) on an independent dataset called DNAiset, which consists of 80
DNA-BPs and 192 non-binding proteins. The featureselection-based model correctly predicted 72 and 170 out of 80 DNA-BPs and 192 non-binding proteins respectively, while the ensemble-based model correctly predicted 68 and 166 out of 80 DNA-BPs and 192 non-binding proteins respectively. This demonstrates that our SVM models perform equally well on the independent dataset.

\section{Performance on realistic dataset}

In a real-world situation, the number of non-binding proteins is significantly higher than DNA-BPs. Thus, it is important to build and evaluate SVM models on more realistic data rather than equal number of DNA-BPs and non-binding proteins. Hence, we obtained a realistic dataset (DNArset), which has 231 DNA-BPs and 1500 non-binding proteins. Firstly, we developed SVM model using feature selection based method on DNArset and achieved the maximum MCC of 0.720 with an accuracy of $94.16 \%$. Then we also developed ensemble learning models and the ensemble stacking method which achieved the maximum MCC of 0.729 with an accuracy of $94.28 \%$, while the majority voting method has a significant poorer performance with more negative proteins which demonstrates the instability of it. The detailed five fold cross validation results and mRMR-IFS results are shown

Table 4 The performance of feature-selection method and ensemble learning

\begin{tabular}{|c|c|c|c|c|c|c|c|c|c|c|c|c|}
\hline \multirow[t]{2}{*}{ Method } & \multicolumn{6}{|c|}{ DNAdset } & \multicolumn{6}{|c|}{ DNAaset } \\
\hline & Acc & AUC & F-score & Sen & $\mathrm{Sp}$ & MCC & Acc & AUC & F-score & Sen & $\mathrm{Sp}$ & MCC \\
\hline mRMR-IFS & 0.940 & 0.973 & 0.940 & 0.964 & 0.917 & 0.881 & 0.789 & 0.864 & 0.793 & 0.819 & 0.766 & 0.575 \\
\hline Ensemble-voting & 0.898 & N/A & 0.900 & 0.905 & 0.892 & 0.797 & 0.789 & $\mathrm{~N} / \mathrm{A}$ & 0.792 & 0.801 & 0.778 & 0.579 \\
\hline Ensemble-stacking & 0.907 & 0.965 & 0.910 & 0.935 & 0.878 & 0.819 & 0.811 & 0.885 & 0.808 & 0.814 & 0.799 & 0.614 \\
\hline
\end{tabular}


Table 5 Comparison of the predicted results by our method and some web-servers on DNAiset

\begin{tabular}{lccccc}
\hline Method & Acc & F-score & Sen & Sp & MCC \\
\hline DNA-Binder & 0.717 & 0.642 & 0.863 & 0.656 & 0.473 \\
DNABIND & 0.842 & 0.739 & 0.763 & 0.875 & 0.627 \\
iDNA-Prot & 0.875 & 0.798 & 0.837 & 0.891 & 0.709 \\
Our method & 0.890 & 0.828 & 0.900 & 0.886 & 0.753 \\
\hline
\end{tabular}

in Additional File 4. Lastly, we applied models trained by DNArset with feature selection method on DNAiset which can correctly predict 60 out of 80 positives and 172 out of 192 negatives (see Additional file 4). The results further confirmed the prediction effectiveness of our method.

\section{Comparison with existing methods}

It is important to compare the performance of our protocol with existing methods in order to evaluate its effectiveness. The performance of feature-selection based models and ensemble learning models for DNA-binding prediction by fivefold cross-validation test is summarized in Table 4 . The feature-selection based protocol has an accuracy of 0.940 and 0.789 for DNAdset and DNAaset respectively, accordingly ensemble learning protocol returns a little lower accuracy of 0.907 for DNAdset but a higher accuracy of 0.811 for DNAaset. For DNAaset, the performances of previously reported studies developed from it are [10], with accuracy of $0.742, \mathrm{MCC}$ of 0.49 , Sen of 0.735 and Sp of 0.749 and [17], with accuracy of 0.755 , MCC of 0.51 , Sen of 0.820 and Sp of 0.690 . As shown in Table 4, the best performance in our protocol with an accuracy of 0.811, MCC of 0.614 , Sen of 0.814 and Sp of 0.799 is much better.
As mentioned above, the DNAdset is a union of three datasets used in previously related studies, it has difficulty in direct comparison. So we adopted the newly developed independent test set called DNAiset to compare our method with several famous web-based servers. Our method correctly predicted 72 out of 80 positives and 170 out of 192 negatives. DNAbinder developed by Kumar et al. [10] can correctly predict 69 and 126 out of 80 DNA-BPs and 192 non-binding proteins respectively for DNAiset (the SVM model trained on main dataset was used). DNABIND developed by Szilagyi et al. [65] which correctly predicts 61 and 168 out of 80 DNA-BPs and 192 non-binding proteins respectively for DNAiset. The iDNA-Prot [12], adopted to select the DNAiset in this study, can correctly predict 67 out of 80 DNA-BPs and 171 out of 192 negatives. It should be noticed that the 192 non-binding proteins in DNAiset were used as training set in this method. Detailed comparison results of different methods can be found in Table 5. It demonstrates that both of our mRMR-IFS feature-selection method and ensemble learning strategy return satisfactory results and outperform some previous studies.

\section{Discussion}

As described in the Methods section, there were four kinds of features transformed by three different methods which produced totally twelve types of feature vectors in this study. For both datasets, the original feature space of 2040 dimensions is reduced to 203 and 153 dimensions separately after mRMR-IFS feature selection process. The distribution of the number of each type of features in the optimal feature set is investigated and shown in Figure 6.

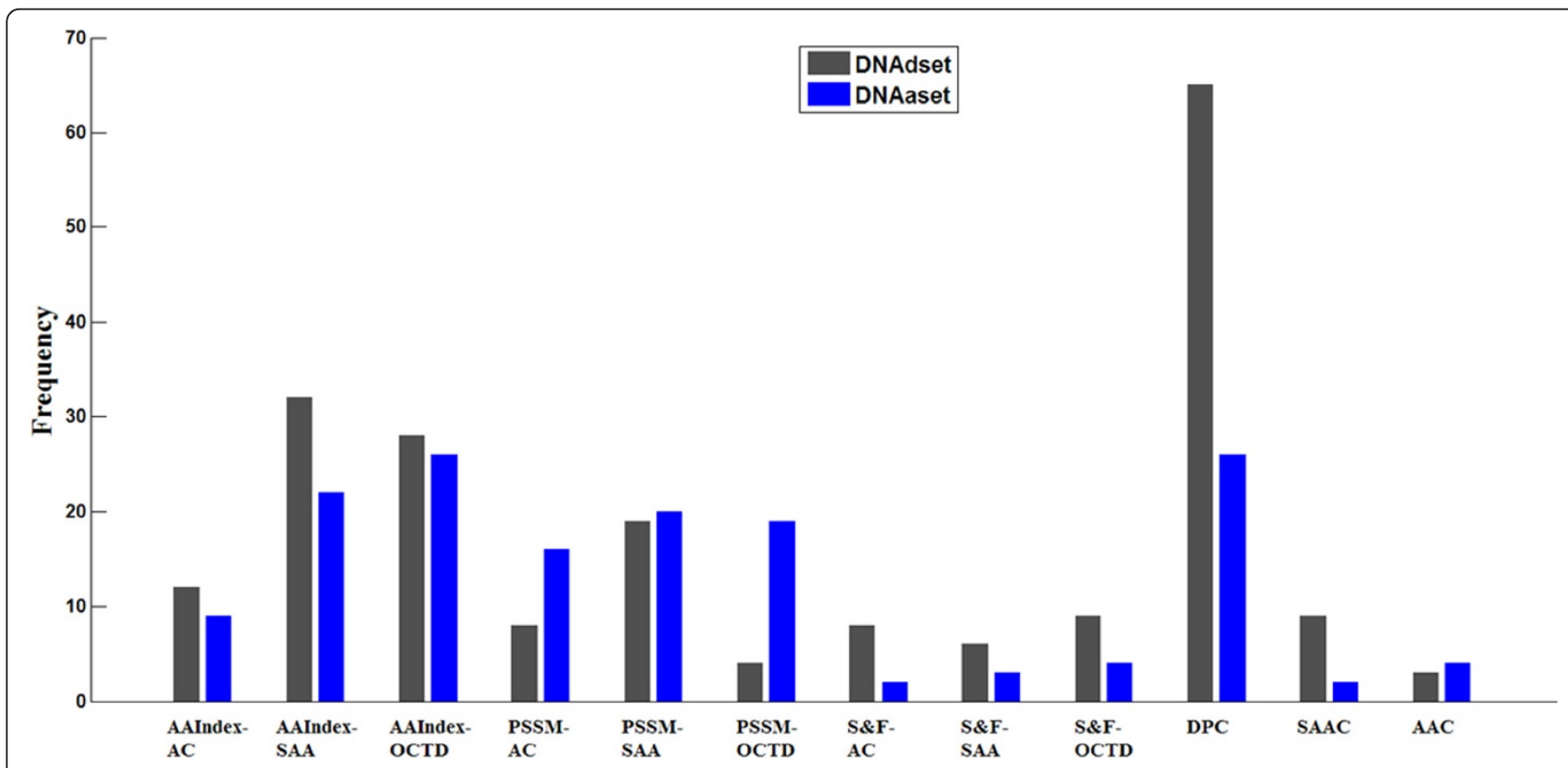

Figure 6 Distribution of the number of each type of features (a total 12 types) in the optimal feature set. 
As we can see from Figure 6, the proportion of each type of feature is similar between DNAdset (with 203 optimal features) and DNAaset (with 153 optimal features) except PSSM-OCTD features. Features of DPC, AAIndex features transformed by SAA and OCTD method and PSSM scores transformed by SAA method are over half of the optimal features and DPC is the highest. After further inspection of DPC in the optimal features we find positively charged lysine or arginine or aromatic phenylalanine most frequently appeared in these optimal two-tuples. These three kinds of amino acids play an important role in protein-DNA interactions as described in previous studies $[66,67]$. The other eight kinds of features like AAIndexAC, PSSM-AC, etc. are also involved in the optimal features. This phenomenon suggests that all twelve kinds of features contribute to the prediction of protein-DNA interactions and that DPC features may play an irreplaceable role for DNA-BPs prediction. Further analysis in DNAdset reveals that physicochemical property and evolutionary information are better represented as local descriptors (by SAA transformation method), while DPC as nonlocal features of composition information is more befitting than others, and the structural/functional property tend to be globally and nonlocally represented (by OCTD and AC method). The same situation can be found in DNAaset, in addition, physicochemical property and evolutionary information transformed by OCTD are equal to SAA, and this isn't hard to understand because DNAdset consists of partial sequences (binding regions or DNA binding domains) while DNAaset consists of full length. Thus global features for both AAIndex and PSSM transformed by OCTD in DNAdset are not really global like DNAaset. The results confirm that different properties of amino acid are preferentially distributed in various scales [32]. On the whole, no single type of feature could undertake the task of DNA-BPs prediction accurately. The most important challenge is to find a suitable way to fully describe the information of protein which is just what we are trying to do.

\section{Conclusions}

In this work, we investigate the idea of ensemble of informative features from different levels for predicting DNA-BPs which is motivated by a recently research result that amino acid physical properties can fall into distinct levels [32]. The overall protocol is aimed at representing the four important kinds of properties of protein appropriately by different transformation methods and seeking the optimal feature descriptors for presentation of DNA-BPs. The performances of individual modules indicate the usefulness of features from various levels and their dissimilarity. Based on the obtained different kinds of feature descriptors, we take two strategies for the construction of the final prediction models which are mRMR-IFS feature selection protocol and ensemble learning approach. Encouragingly, we get good performance of Acc of 0.940 for DNAdset with the mRMR-IFS method and Acc of 0.811 for DNAaset with ensemble learning approach, and the performance on independent test set is also good.

Our experiments indicate that it may be helpful to develop a successful machine method to predict the DNABPs by exploiting protein sequence comprehensively. However more explorations about amino acid properties are still needed in this direction and further work on interpreting these features and exploring mechanisms of protein-DNA interactions are underway.

\section{Additional files}

Additional file 1: Complete list of PDB codes for DNAdset and DNAiset.

Additional file 2: The IFS results for DNAdset.

Additional file 3: The IFS results for DNAaset.

Additional file 4: The IFS results and model evaluation for DNArset.

\section{Competing interests}

The authors declare that they have no competing interests.

\section{Authors' contributions}

$\mathrm{HL}$ conceived the study, reviewed and revised the manuscript. CZ developed the methods, performed the analysis and drafted the manuscript. JG supervised the whole project and participated in manuscript preparation. All authors have read and approved the final manuscript.

\section{Authors' information}

Correspondence and requests for reprints to:

Prof. Honglin Li

School of Pharmacy, East China University of Science and Technology 130

Mei Long Road, Shanghai 200237

Phone/Fax: +86-21-64250213

\section{Acknowledgments}

This work was supported by the Fundamental Research Funds for the Central Universities, the National Natural Science Foundation of China (grants 21173076, 81102375, 81230090, 81222046 and 81230076), the Special Fund for Major State Basic Research Project (grant 2009CB918501), the Shanghai Committee of Science and Technology (grant 11DZ2260600), and the 863 $\mathrm{Hi}$-Tech Program of China (grant 2012AA020308). Honglin Li is also sponsored by Program for New Century Excellent Talents in University (grant NCET-10-0378).

Received: 29 September 2012 Accepted: 4 March 2013

Published: 9 March 2013

\section{References}

1. Luscombe NM, Austin SE, Berman HM, Thornton JM: An overview of the structures of protein-DNA complexes. Genome Biol 2000, 1(1):1-37.

2. Ren B, Robert F, Wyrick JJ, Aparicio O, Jennings EG, Simon I, Zeitlinger J, Schreiber J, Hannett N, Kanin E, et al: Genome-wide location and function of DNA binding proteins. Science 2000, 290(5500):2306-2309.

3. Ahmad S, Sarai A: Moment-based prediction of DNA-binding proteins. J Mol Biol 2004, 341(1):65-71.

4. Zhao H, Yang Y, Zhou Y: Structure-based prediction of DNA-binding proteins by structural alignment and a volume-fraction corrected DFIREbased energy function. Bioinformatics 2010, 26(15):1857-1863.

5. Tjong $H$, Zhou HX: DISPLAR: an accurate method for predicting DNAbinding sites on protein surfaces. Nucleic Acids Res 2007, 35(5):1465-1477.

6. Stawiski EW, Gregoret LM, Mandel-Gutfreund Y: Annotating nucleic acidbinding function based on protein structure. J Mol Biol 2003, 326(4):1065-1079. 
7. Cai YD, Lin SL: Support vector machines for predicting rRNA-, RNA-, and DNA-binding proteins from amino acid sequence. Biochim Biophys Acta 2003, 1648(1-2):127-133.

8. Fang $Y$, Guo $Y$, Feng $Y$, Li M: Predicting DNA-binding proteins: approached from Chou's pseudo amino acid composition and other specific sequence features. Amino Acids 2008, 34(1):103-109.

9. Gao M, Skolnick J: A threading-based method for the prediction of DNAbinding proteins with application to the human genome. PLoS Comput Biol 2009, 5(11):e1000567.

10. Kumar M, Gromiha M, Raghava G: Identification of DNA-binding proteins using support vector machines and evolutionary profiles. BMC Bioinforma 2007, 8(1):463.

11. Shao X, Tian $Y$, Wu L, Wang $Y$, Jing $L$, Deng N: Predicting DNA- and RNAbinding proteins from sequences with kernel methods. J Theor Biol 2009, 258(2):289-293.

12. Lin WZ, Fang JA, Xiao X, Chou KC: IDNA-prot: identification of DNA binding proteins using random forest with grey model. PLoS One 2011, 6(9):e24756

13. Cai YD, Doig AJ: Prediction of Saccharomyces cerevisiae protein functional class from functional domain composition. Bioinformatics 2004, 20(8):1292-1300.

14. Brameier M, Haan J, Krings A, MacCallum R: Automatic discovery of cross-family sequence features associated with protein function. BMC Bioinforma 2006, 7(1):16.

15. Brown J, Akutsu T: Identification of novel DNA repair proteins via primary sequence, secondary structure, and homology. BMC Bioinforma 2009, 10(1):25.

16. Bhardwaj N, Langlois RE, Zhao G, Lu H: Kernel-based machine learning protocol for predicting DNA-binding proteins. Nucleic Acids Res 2005, 33(20):6486-6493.

17. Huang HL, Lin IC, Liou YF, Tsai CT, Hsu KT, Huang WL, Ho SJ, Ho SY: Predicting and analyzing DNA-binding domains using a systematic approach to identifying a set of informative physicochemical and biochemical properties. BMC Bioinforma 2011, 12(Suppl 1):S47.

18. Xiong Y, Liu J, Wei DQ: An accurate feature-based method for identifying DNA-binding residues on protein surfaces. Proteins 2011, 79(2):509-517.

19. Ahmad S, Andrabi M, Mizuguchi K, Sarai A: Prediction of mono- and dinucleotide-specific DNA-binding sites in proteins using neural networks. BMC Struct Biol 2009, 9:30.

20. Nimrod G, Schushan M, Szilágyi A, Leslie C, Ben-Tal N: iDBPs: a web server for the identification of DNA binding proteins. Bioinformatics 2010, 26(5):692-693

21. Yan C, Terribilini M, Wu F, Jernigan R, Dobbs D, Honavar V: Predicting DNA-binding sites of proteins from amino acid sequence. BMC Bioinforma 2006, 7(1):262

22. Govindan G, Nair AS: New Feature Vector for Apoptosis Protein Subcellular Localization Prediction. In Advances in Computing and Communications Communications. Volume 190. Edited by Abraham A Kochi: Springer Berlin Heidelberg; 2011:294-301.

23. Qian ZL, Cai YD, Li YX: A novel computational method to predict transcription factor DNA binding preference. Biochem Biophys Res Commun 2006, 348(3):1034-1037.

24. Nanni L, Lumini A: Combing ontologies and dipeptide composition for predicting DNA-binding proteins. Amino Acids 2008, 34(4):635-641.

25. Xia JF, Zhao XM, Huang DS: Predicting protein-protein interactions from protein sequences using meta predictor. Amino Acids 2010, 39(5):1595-1599.

26. Liu ZP, Wu LY, Wang Y, Zhang XS, Chen LN: Bridging protein local structures and protein functions. Amino Acids 2008, 35(3):627-650.

27. Chou KC: Some remarks on protein attribute prediction and pseudo amino acid composition. J Theor Biol 2011, 273(1):236-247.

28. Yuan Y, Shi X, Li X, Lu W, Cai Y, Gu L, Liu L, Li M, Kong X, Xing M: Prediction of interactiveness of proteins and nucleic acids based on feature selections. Mol Divers 2010, 14(4):627-633.

29. Song J, Tan H, Takemoto K, Akutsu T: HSEpred: predict half-sphere exposure from protein sequences. Bioinformatics 2008, 24(13):1489-1497.

30. Nanni L, Brahnam S, Lumini A: High performance set of PseAAC and sequence based descriptors for protein classification. J Theor Biol 2010, 266(1):1-10.

31. Zhang Z, Kochhar S, Grigorov MG: Descriptor-based protein remote homology identification. Protein Sci 2005, 14(2):431-444.

32. Rackovsky S: Global characteristics of protein sequences and their implications. Proc Natl Acad Sci USA 2010, 107(19):8623-8626.
33. Huang Y, Niu B, Gao Y, Fu L, Li W: CD-HIT Suite: a web server for clustering and comparing biological sequences. Bioinformatics 2010, 26(5):680-682.

34. Wang G, Dunbrack RL Jr: PISCES: a protein sequence culling server. Bioinformatics 2003, 19(12):1589-1591.

35. Chawla NV, Japkowicz N, Kotcz A: Editorial: special issue on learning from imbalanced data sets. SIGKDD Explor News/ 2004, 6(1):1-6.

36. Chang CC, Lin CJ: LIBSVM: A library for support vector machines. ACM Transact Intell Syst Technol 2011, 2(3):27.

37. Feng ZP: Prediction of the subcellular location of prokaryotic proteins based on a new representation of the amino acid composition. Biopolymers 2001, 58(5):491-499.

38. Bhasin M, Raghava GPS: ESLpred: SVM-based method for subcellular localization of eukaryotic proteins using dipeptide composition and PSI-BLAST. Nucleic Acids Res 2004, 32(suppl 2):W414-W419.

39. Peng $H$, Long $F$, Ding $C$ : Feature selection based on mutual information: criteria of max-dependency, max-relevance, and min-redundancy. IEEE Trans Pattern Anal Mach Intell 2005, 27(8):1226-1238.

40. Tantoso E, Li KB: AAlndexLoc: predicting subcellular localization of proteins based on a new representation of sequences using amino acid indices. Amino Acids 2008, 35(2):345-353.

41. Afridi T, Khan A, Lee Y: Mito-GSAAC: mitochondria prediction using genetic ensemble classifier and split amino acid composition. Amino Acids 2012, 42(4):1443-1454

42. Han $P$, Zhang $X$, Feng Z-P: Predicting disordered regions in proteins using the profiles of amino acid indices. BMC Bioinforma 2009, 10(Suppl 1):S42.

43. Kawashima S, Pokarowski P, Pokarowska M, Kolinski A, Katayama T, Kanehisa M: AAindex: amino acid index database, progress report 2008. Nucleic Acids Res 2008, 36(Database issue):D202-205.

44. Schaffer AA, Aravind L, Madden TL, Shavirin S, Spouge JL, Wolf YI, Koonin EV, Altschul SF: Improving the accuracy of PSI-BLAST protein database searches with composition-based statistics and other refinements. Nucleic Acids Res 2001, 29(14):2994-3005.

45. Chen SA, Ou YY, Lee TY, Gromiha MM: Prediction of transporter targets using efficient RBF networks with PSSM profiles and biochemical properties. Bioinformatics 2011, 27(15):2062-2067.

46. Song J, Tan H, Wang M, Webb Gl, Akutsu T: TANGLE: Two-level support vector regression approach for protein backbone torsion angle prediction from primary sequences. PLoS One 2012, 7(2):e30361.

47. Chu WY, Huang YF, Huang CC, Cheng YS, Huang CK, Oyang YJ: ProteDNA: a sequence-based predictor of sequence-specific DNA-binding residues in transcription factors. Nucleic Acids Res 2009, 37(suppl 2):W396-W401.

48. Jones DT: Protein secondary structure prediction based on positionspecific scoring matrices. J Mol Biol 1999, 292(2):195-202.

49. Wright $P E$, Dyson HJ: Intrinsically unstructured proteins: re-assessing the protein structure-function paradigm. J Mol Biol 1999, 293(2):321-331.

50. Lobley A, Swindells MB, Orengo CA, Jones DT: Inferring function using patterns of native disorder in proteins. PLoS Comput Biol 2007, 3(8):e162.

51. Weiss MA, Ellenberger T, Wobbe CR, Lee JP, Harrison SC, Struhl K: Folding transition in the DNA-binding domain of GCN4 on specific binding to DNA. Nature 1990, 347(6293):575-578.

52. Dosztányi Z, Csizmok V, Tompa P, Simon I: IUPred: web server for the prediction of intrinsically unstructured regions of proteins based on estimated energy content. Bioinformatics 2005, 21(16):3433-3434.

53. Dubchak I, Muchnik I, Holbrook SR, Kim SH: Prediction of protein folding class using global description of amino acid sequence. Proc Natl Acad Sci USA 1995, 92(19):8700-8704

54. Govindan G, Nair AS: Composition, Transition and Distribution (CTD) - A dynamic feature for predictions based on hierarchical structure of cellular sorting. Hyderabad: India Conference (INDICON); 2011. 2011 Annual IEEE; 16-18 Dec

55. Cai CZ, Han LY, Ji ZL, Chen X, Chen YZ: SVM-Prot: Web-based support vector machine software for functional classification of a protein from its primary sequence. Nucleic Acids Res 2003, 31(13):3692-3697.

56. Wold S, Jonsson J, Sjörström M, Sandberg M, Rännar S: DNA and peptide sequences and chemical processes multivariately modelled by principal component analysis and partial least-squares projections to latent structures. Anal Chim Acta 1993, 277(2):239-253.

57. Doytchinova IA, Flower DR: VaxiJen: a server for prediction of protective antigens, tumour antigens and subunit vaccines. BMC Bioinforma 2007, 8:4

58. Guo Y, Yu L, Wen Z, Li M: Using support vector machine combined with auto covariance to predict protein-protein interactions from protein sequences. Nucleic Acids Res 2008, 36(9):3025-3030. 
59. Lee BJ, Shin MS, Oh YJ, Oh HS, Ryu KH: Identification of protein functions using a machine-learning approach based on sequence-derived properties. Proteome science 2009, 7:27.

60. Yang L, Xia JF, Gui J: Prediction of protein-protein interactions from protein sequence using local descriptors. Protein Pept Lett 2010, 17(9):1085-1090.

61. Davies MN, Secker A, Freitas AA, Clark E, Timmis J, Flower DR: Optimizing amino acid groupings for GPCR classification. Bioinformatics 2008, 24(18):1980-1986,

62. Si J, Zhang Z, Lin B, Schroeder M, Huang B: MetaDBSite: a meta approach to improve protein DNA-binding sites prediction. BMC Syst Biol 2011, 5(Suppl 1):S7.

63. Rokach L: Ensemble-based classifiers. Artif Intell Rev 2010, 33(1):1-39.

64. Sathyapriya R, Vijayabaskar MS, Vishveshwara S: Insights into Protein-DNA Interactions through structure network analysis. PLoS Comput Biol 2008, 4(9):e1000170

65. Szilagyi A, Skolnick J: Efficient prediction of nucleic acid binding function from low-resolution protein structures. J Mol Biol 2006, 358(3):922-933.

66. Ghosh S, Marintcheva B, Takahashi M, Richardson CC: C-terminal phenylalanine of bacteriophage $\mathrm{T7}$ single-stranded DNA-binding protein is essential for strand displacement synthesis by T7 DNA polymerase at a nick in DNA. J Biol Chem 2009, 284(44):30339-30349.

67. Rohs R, West SM, Sosinsky A, Liu P, Mann RS, Honig B: The role of DNA shape in protein-DNA recognition. Nature 2009, 461(7268):1248-1253.

\section{doi:10.1186/1471-2105-14-90}

Cite this article as: Zou et al:: An improved sequence based prediction protocol for DNA-binding proteins using SVM and comprehensive feature analysis. BMC Bioinformatics 2013 14:90.

\section{Submit your next manuscript to BioMed Central and take full advantage of:}

- Convenient online submission

- Thorough peer review

- No space constraints or color figure charges

- Immediate publication on acceptance

- Inclusion in PubMed, CAS, Scopus and Google Scholar

- Research which is freely available for redistribution 